continued throughout the experiment. We also tested mice lacking the toll-like receptor adaptor protein, MyD88. Cognitive abilities were tested using spontaneous alternation in a Y-maze. RESULTS/ ANTICIPATED RESULTS: We found that minocycline reduces fatigue-like behavior exhibited after irradiation, but had no effect on pre-irradiation activity levels. Similarly, fatigue-like behavior after radiation was partially reversed by genetic loss of MyD88. Y-maze spontaneous alternation performance remained similar in all groups. DISCUSSION/SIGNIFICANCE OF IMPACT: Both pharmacological and genetic anti-inflammatory manipulations increased voluntary activity levels after irradiation. Our results suggest that inflammation is an important factor in the development of fatigue-like behavior. Modulators of inflammatory processes hold potential for alleviating fatigue.

4361

\section{Interim Results of the Effect of Haptoglobin Phenotype on Inflammatory Cytokine Concentrations in Plasma and CSF After Aneurysmal Subarachnoid Hemorrhage \\ Austin Smith ${ }^{1}$ \\ ${ }^{1}$ University of Kentucky Center for Clinical and Translational Science}

OBJECTIVES/GOALS: Haptoglobin (Hp) phenotypes may affect inflammatory response after neurologic injury. We are investigating the relationship between patient $\mathrm{Hp}$ phenotypes and inflammatory cytokine concentrations in plasma and CSF after aneurysmal subarachnoid hemorrhage (aSAH), a severe form of hemorrhagic stroke. METHODS/STUDY POPULATION: Following IRB approval, all patients with angiographically-proven aSAH and who underwent extraventricular drain (EVD) placement were included. Patients were excluded if they were not expected to survive hospitalization or were on pre-existing anti-inflammatory agents. For all enrolled patients, plasma and CSF samples were taken on post-bleed days $3,5,7$, and 10 , processed and then frozen for later analysis. In this interim analysis, Hp phenotype was assessed through ELISA analysis of plasma samples and cytokine concentrations were determined using multiplex ELISA kits for both plasma and CSF samples. Hp phenotypes were dichotomized to either HP1 (Hp 1-1 or 1-2) or HP2 (Hp 2-2). RESULTS/ANTICIPATED RESULTS: To date, 23 aSAH patients have been enrolled in this IRB-approved study. An interim analysis of the first 13 patients has revealed eight Hp1 patients (Hp 1-1 n = 1, Hp 1-2 n=7) and five Hp2 patients. CSF levels of IL-6 and TNF-a were greater than plasma levels in all patients at all time points. CSF levels of IL-6 appear to peak on PBD $5(1890 \pm 767 \mathrm{pcg} / \mathrm{mL})$ and $7(1612 \pm 899 \mathrm{pcg} / \mathrm{mL})$. The CSF IL-6 concentrations in the Hp2 group were lower (1792 \pm $806 \mathrm{pcg} / \mathrm{mL}$ vs. $1952 \pm 791 \mathrm{pcg} / \mathrm{mL})$ on PBD5 but were higher $(1635 \pm 930 \mathrm{pcg} / \mathrm{mL}$ vs $1598 \pm 943 \mathrm{pcg} / \mathrm{mL})$ at PBD7; however, these differences did not reach statistical significance. DISCUSSION/ SIGNIFICANCE OF IMPACT: This interim analysis demonstrated no statistically significant differences in plasma or CSF cytokine concentrations between patients with different $\mathrm{Hp}$ phenotypes. This may be due to the low number of samples or the potential confounding effect of disease-specific secondary neurological injuries.
4126

Intermuscular adipose tissue secretes pro-inflammatory, extracellular matrix, and lipid signals related to insulin resistance and type 2 diabetes

Darcy Kahn ${ }^{1}$, Simona Zarini ${ }^{2}$, Emily Macias ${ }^{2}$, Amanda Garfield ${ }^{2}$, Kathleen Harrison ${ }^{2}$, Melanie Cree-Green ${ }^{2}$, Jonathan Schoen ${ }^{2}$, and Bryan Bergman ${ }^{2}$

${ }^{1}$ University of Colorado at Denver; ${ }^{2}$ University of Colorado, Anschutz Medical Campus

OBJECTIVES/GOALS: Intermuscular adipose tissue (IMAT) has been associated with insulin resistance and type 2 diabetes, yet mechanistic studies addressing the functional role of IMAT are lacking. The aim of this work was to identify novel mechanisms by which IMAT may directly impact skeletal muscle metabolism. METHODS/ STUDY POPULATION: We quantified the secretome of IMAT, subcutaneous adipose tissue (SAT), and visceral adipose tissue (VAT) to determine if there are differences between depots in the secretion of cytokines, eicosanoids, FFAs and proteins that influence metabolic function. SAT and VAT biopsies from patients undergoing laparoscopic bariatric surgery and IMAT extracted from vastus lateralis biopsies of individuals with Obesity were cultured for 48 hours in DMEM, and the conditioned media was analyzed using nanoflow HPLC-MS, multiplex ELISAs and LC/MS/MS for proteins, cytokines and eicosanoids/FFA, respectively. RESULTS/ ANTICIPATED RESULTS: IMAT secretion of various extracellular matrix proteins (fibrinogen- $\beta$, collagenV1a3, fibronectin) was significantly different than VAT and SAT. Pro-inflammatory cytokine secretion of IFNg, TNFa, IL-8 and IL-13 from IMAT was higher than VAT and significantly higher than SAT $(p<0.05)$. IMAT secretes significantly more pro-inflammatory eicosanoids $\mathrm{TXB}_{2}$ and $\mathrm{PGE}_{2}$ than VAT $(p=0.02,0.05)$ and SAT $(p=0.01,0.04)$. IMAT and VAT have significantly greater basal lipolysis assessed by FFA release rates compared to SAT $(\mathrm{p}=0.01,0.04)$. DISCUSSION/ SIGNIFICANCE OF IMPACT: These data begin to characterize the disparate secretory properties of SAT, VAT and IMAT and suggest a metabolically adverse secretome of IMAT, that due to its proximity to skeletal muscle may play an important functional role in the pathogenesis of insulin resistance and type 2 diabetes.

4558

Investigating the functional consequences of anaplastic lymphoma kinase (ALK) mutations arising upon Lorlatinib treatment

Gabriela Maria Witek ${ }^{1}$, Whelton Miller, David Slochower, Esther Berko, Yael Mossé, Mark Lemmon, and Ravi Radhakrishnan

${ }^{1}$ University of Pennsylvania School of Medicine

OBJECTIVES/GOALS: Neuroblastoma (NB) is an embryonal cancer of the sympathetic nervous system that affects mostly infants and young children. The complex genetic background present across NB patients results in diverse clinical response and difficulty in individualizing therapy. Currently, NB patients undergo a regimen of genotoxic chemotherapeutics, radiation therapy, and new immunotherapy that, while effective, has significant side effects, including excruciating pain. One promising avenue for targeted therapy in neuroblastoma focuses on anaplastic lymphoma kinase (ALK), a cell surface neural receptor tyrosine kinase. We previously identified 
activating point mutations within the tyrosine kinase domain of ALK as the primary cause of hereditary $\mathrm{NB}$, and we and others subsequently showed that these same alterations are the most common somatic single-nucleotide mutations in the sporadic forms of the disease. Crizotinib, a first-generation small molecule ATP-competitive inhibitor of the ALK tyrosine kinase, showed limited anti-tumor activity in patients with relapsed NB harboring ALK F1174 and F1245 mutations. We have demonstrated that lorlatinib, a novel ATP-competitive ALK inhibitor, overcomes this de novo resistance in preclinical models of ALK-driven NB. Recent clinical trials with lorlatinib in patients with non-small cell lung cancer harboring an ALK fusion, and in patients with NB harboring ALK mutations show the emergence of multiple or compound ALK mutations as a mechanism of resistance. We postulate that these compound mutations disrupt the interaction between and ALK and cause resistance. In this study, we employ a computational approach to model mutated ALK in complex with lorlatinib as well as ATP to understand whether the new mutations alter the affinity or mode of lorlatinib/ATP binding to ALK, and thus cause suboptimal ALK inhibition. METHODS/ STUDY POPULATION: We employ methods in computational structural biology and drug design, primarily based on molecular modeling, molecular dynamics (MD), and molecular docking. Based on existing crystal structures of wildtype ALK, we model the mutations and perform MD simulations in order to characterize the activation state of the protein as well as perform ensemble docking calculations to assess the binding affinities and modes in ALKlorlatinib and ALK-ATP complexes. RESULTS/ANTICIPATED RESULTS: We expect that the compound mutations cause resistance to lorlatinib either by lowering protein affinity for the drug or increasing the affinity for ATP. Alternatively, the compound mutations may disrupt the protein activation state, in which case ALK may no longer be active, and another protein/pathway could be driving the resistance. DISCUSSION/SIGNIFICANCE OF IMPACT: The results of this study will enable the understanding of the mechanism of resistance to lorlatinib and facilitate the design of new ALK inhibitors, or help develop more optimal and mechanism-guided therapies aimed to overcome the resistance.

4040

\section{Investigation of a Series of 1,4-diaryl-pyrazolo-}

\section{pyridinones as Anti-Leishmanial Agents}

Hannah Noel Corman ${ }^{1}$, Douglas A. Shoue ${ }^{2}$, Bruce J. Melancon ${ }^{3}$, and Mary Ann McDowell ${ }^{2}$

${ }^{1}$ University of Notre Dame; ${ }^{2}$ Eck Institute of Global Health, University of Notre Dame; ${ }^{3}$ Warren Center Drug Discovery \& Development, University of Notre Dame

OBJECTIVES/GOALS: This study was conducted in order to identify novel chemical compounds that exhibit anti-leishmanial activity and to further characterize their efficacy and toxicity in in vitro and in vivo systems in the hopes of future chemotherapeutic developments. METHODS/STUDY POPULATION: We developed a novel, target-free fluorometric high-throughput screen (HTS) to identify small molecules with anti-leishmanial activity. Screening of 10,000 small molecules from the ChemBridge DIVERset-EXP library cassette \#5 yielded 210 compounds that killed $80 \%$ of parasites. One hundred nine (109) molecular scaffolds were represented within the hit compounds, including the 1,4-diaryl-pyrazolo-pyridinone (1,4-DAPP). A total of 27 novel 1,4-DAPP compounds were synthesized and anti-leishmanial efficacy and host cell toxicity was determined using L. donovani mCherry expressing amastigotes and THP-1 macrophages. Additional pharmacokinetic analyses of a potent 1,4-DAPP compound were conducted. RESULTS/ ANTICIPATED RESULTS: Four experimental compounds had IC50 values less than $5 \mu \mathrm{M}$, providing similar anti-leishmanial activity to miltefosine. Compound 9279817 had a clearance almost twice the rate of normal hepatic blood flow and had a relatively high volume of distribution, indicating this compound is rapidly cleared and distributes into tissues. in vitro rat liver microsome assays suggest a rapid metabolism of 9279817 , and MS/MS results suggest this metabolite is most likely formed via oxidation of the sulfur on the lower aromatic ring. DISCUSSION/SIGNIFICANCE OF IMPACT: This study revealed a novel structural class of compounds that have anti-leishmanial activity. in vitro experiments show compounds with similar efficacy as miltefosine while having significantly less toxicity, suggesting that this class could be further developed as a potential chemotherapeutic.

4447

\section{Leptin supplementation prevents the loss of hypoglycemia-induced glucagon release following} exposure to six days of severe caloric restriction in mice David H McDougal ${ }^{1}$, Marina A. DuVall, Christopher D. Morrison, Laura A. Moldovan, and Rajvi Jariwala

${ }^{1}$ Pennington Biomedical Research Center- LA CaTS

OBJECTIVES/GOALS: We have recently shown that mice exposed to six days of $60 \%$ caloric restriction acutely display reduced hypoglycemia-induced glucagon release following refeeding, and that this effect is concurrent with low leptin levels. The current study was conducted to ascertain if leptin treatment during caloric restriction would reverse this effect. METHODS/STUDY POPULATION: Three groups of mice were used, an ad libitum (Ad-lib) fed group and two caloric restriction (CR) groups, one of which received twice daily leptin injection $(0.5-1 \mu \mathrm{g} / \mathrm{g}$; IP) and the other vehicle (saline) during their caloric restriction. CR mice were placed on $60 \%$ caloric restriction for 6 consecutive days. Ad lib mice were housed in an identical manner but fed ad libitum during this same period. Following 6 days of restriction, CR mice were given ad lib access to food for $16 \mathrm{~h}$. After the $16 \mathrm{~h}$ period of refeeding, both CR and ad lib mice began a $6 \mathrm{~h}$ fast which was immediately followed by a hypoglycemic insulin tolerance test (ITT). ITTs consisted of a variable dose of insulin intended to achieve a blood glucose of $\sim 45 \mathrm{mg} / \mathrm{dL}$ within 60 minutes, at which time blood was collected for glucagon and corticosterone assays. RESULTS/ANTICIPATED RESULTS: The mean blood glucose levels during the ITT at 45 and 60 minutes post injection across all three groups were $46.8+3.1$ and $37.0+2.4$, respectively. There were no significant differences in glucose levels between the three groups at these two time points. As expected, saline treated CR mice displayed significantly reduced serum glucagon levels in response to the ITT relative to Ad-lib mice $(23.5+10.9$ vs. $91.7+20.8 \mathrm{pg} / \mathrm{mL}, \mathrm{p}=0.009)$. In contrast, leptin-treated CR mice maintained their hypoglycemia-induced glucagon response to the ITT $(78.0+16.8 \mathrm{pg} / \mathrm{mL}, \mathrm{p}>0.99$ vs. Ad-lib group). In addition, although corticosterone levels in saline treated $\mathrm{CR}$ mice were numerically lower than in Ad-lib mice, this difference was not statistically significance $(3928+277$ vs. $4571+178 \mathrm{pg} / \mathrm{mL}, \mathrm{p}=0.179)$. DISCUSSION/SIGNIFICANCE OF IMPACT: Diabetes patients on insulin therapy often develop impaired hypoglycemic counter-regulation which can lead to life-threatening hypoglycemic complications. Our results suggest that leptin may hold promise as a 